\title{
Patterns of multiple health risk-behaviours in university students and their association with mental health: application of latent class analysis
}

\author{
M. Y. Kwan, PhD (1,2); K. P. Arbour-Nicitopoulos, PhD (3); E. Duku, PhD (4); G. Faulkner, PhD (3,5)
}

This article has been peer reviewed.

Tweet this article

\begin{abstract}
Introduction: University and college campuses may be the last setting where it is possible to comprehensively address the health of a large proportion of the young adult population. It is important that health promoters understand the collective challenges students are facing, and to better understand the broader lifestyle behavioural patterning evident during this life stage. The purpose of this study was to examine the clustering of modifiable health-risk behaviours and to explore the relationship between these identified clusters and mental health outcomes among a large Canadian university sample.
\end{abstract}

Methods: Undergraduate students $(n=837$; mean age $=21$ years $)$ from the University of Toronto completed the National College Health Assessment survey. The survey consists of approximately 300 items, including assessments of student health status, mental health and health-risk behaviours. Latent class analysis was used to identify patterning based on eight salient health-risk behaviours (marijuana use, other illicit drug use, risky sex, smoking, binge drinking, poor diet, physical inactivity, and insufficient sleep).

Results: A three-class model based on student behavioural patterns emerged: "typical," "high-risk" and "moderately healthy." Results also found high-risk students reporting significantly higher levels of stress than typical students $\left(\chi^{2}(1671)=7.26, p<.01\right)$.

Conclusion: Students with the highest likelihood of engaging in multiple health-risk behaviours reported poorer mental health, particularly as it relates to stress. Although these findings should be interpreted with caution due to the $28 \%$ response rate, they do suggest that interventions targeting specific student groups with similar patterning of multiple health-risk behaviours may be needed.

Keywords: university students, mental health, health-risk behaviours, latent class analysis

\section{Introduction}

Behaviours that students acquire or reinforce while in college and university may shape their future health and the health of future generations through the students' eventual roles as parents and leaders. With approximately 20 million enrolled in over 6000 postsecondary institutions across the United States and Canada, ${ }^{1}$ their influence on health could be significant.

\section{Highlights}

- This study examined modifiable health-risk behaviours and mental health outcomes among Canadian undergraduate students.

- There are three discernable patterns of health-risk behaviours among these students.

- Our findings support the notion that engaging in multiple health-risk behaviours is associated with poorer mental health among the university student population.

- Interventions targeting specific student groups with similar patterning of multiple health-risk behaviours may be needed, and a greater emphasis should be placed on supporting health-protecting behaviours of diet, physical activity, and sleep.

The 2015 Okanagan Charter for Health Promoting Universities and Colleges is an international charter developed to promote health in university and college students. The Charter recognizes that postsecondary institutions must focus on health promotion and that the campus is an ideal setting for health-promoting initiatives. ${ }^{2}$ Because campuses often have subsidized facilities, programs and staff who support interventions or health-promoting work, postsecondary students can benefit from sustained health messaging.

Author references:

1. Department of Family Medicine, McMaster University, Hamilton, Ontario, Canada

2. Michael G. DeGroote School of Medicine, Niagara Regional Campus, McMaster University, St. Catharines, Ontario, Canada

3. Faculty of Kinesiology and Physical Education, University of Toronto, Toronto, Ontario, Canada

4. Department of Psychiatry and Behavioural Neurosciences and Offord Centre for Child Studies, McMaster University, Hamilton, Ontario, Canada

5. School of Kinesiology, University of British Columbia, Vancouver, British Columbia, Canada

Correspondence: Matthew Kwan, Department of Family Medicine, 100 Main Street, DBHSC $5^{\text {th }}$ Floor, Hamilton, ON L8P 1H6; Tel: 905-525-9140 ext. 28412; Fax: 905-527-4440; Email: kwanmy@mcmaster.ca 
High school graduation is largely considered to be the first major life transition, as it is accompanied by substantial adjustments across several life domains. ${ }^{3}$ Health promotion during this period is particularly salient because a substantial proportion of the population starts to engage in health-risk behaviours. ${ }^{4}$ Emerging adulthood is a time of significant increases in tobacco, marijuana and alcohol use and decreases in health-protective behaviours such as physical activity and healthy eating.,5 Given younger adults' greater autonomy, we can expect changes in behaviour: Previous inhibitions about health-risk behaviours may weaken with reduced adult supervision and an increased perception that many health-risk behaviours are normative adult behaviours. ${ }^{6}$ Postsecondary institutions, with large numbers of young adults converged into this shared space, may be the last setting to comprehensively address the health of a relatively self-contained population.

While it is likely that many health-risk behaviours are interrelated, most epidemiological research into health behaviours in the postsecondary student population have failed to consider the potential of such interrelationships; ${ }^{7}$ studies typically examine multiple healthrisk behaviours as separate dependent measures. $^{8}$ It is important that health promoters understand the collective challenges students face. In addition, researchers should understand the broader lifestyle behavioural patterning evident during this life stage.

Emerging statistical techniques such as latent class analysis (LCA) allow multiple health-risk behaviours or multiple dependent variables to be investigated together. Specifically, LCA identifies a parsimonious number of classes of individuals who display similar responses. ${ }^{9}$ Segmenting populations into specific groups based on behavioural profiles may improve the scope, utilization and efficacy of interventions that target multiple modifiable health-risk behaviours simultaneously. ${ }^{10}$

Laska et al $^{11}$ published the first study to use LCA to examine lifestyle patterning of modifiable health-risk behaviours among postsecondary students. They found four distinct behavioural patterns, information that may be useful for tailoring on-campus health promotion activities to target these specific student segments. However, little is known how such behavioural typologies (i.e. shared patterning of health-risk behaviours) may be related to mental health outcomes such as stress, anxiety and depression.

Postsecondary students' psychological well-being is increasingly recognized as important. ${ }^{2,7}$ Young adults appear to experience greater stress and depressive symptoms than in the past, ${ }^{12}$ and have higher levels of psychological distress than the general population. ${ }^{13}$ Examining the relationship between multiple health-risk behaviours and mental health outcomes may help determine the behavioural profiles of postsecondary students and how these relate to their mental health.

The primary purpose of this study was to use LCA to examine the distinct health-risk behavioural patterns in a sample of Canadian university students. Advancing the work of Laska and colleagues, ${ }^{11}$ we also examined the relationship between the identified latent classes and several mental health outcomes including fatigue, stress, depression, anxiety and psychological distress.

\section{Methods}

\section{Database and sample}

We collected data during the spring of 2009 using the National College Health Assessment (NCHA). ${ }^{14}$ (For further information, see the American College Health Association [ACHA] website ${ }^{15}$.) The NCHA survey, which has been evaluated extensively for reliability and validity among college and university students in the United States, ${ }^{15}$ consists of approximately 300 questions, including assessments of student health status, mental health and health behaviours.

To obtain our study sample, 5000 students from the approximately 50000 on the St. George campus at the University of Toronto were randomly chosen to participate in the survey. Each student was emailed an invitation to complete the web-based survey on a secure website maintained by the ACHA. Three reminders to complete the online survey were sent to the students over one month.

Our sample comprised 837 full-time undergraduate students (survey response rate $=27.9 \%$ ). The mean age (standard deviation) of the sample was 20.92 (3.73) years, and participants were predominantly female (64\%) and Caucasian $(65 \%)$. The characteristics of our sample are shown in Table 1.

Study procedures were approved by the University of Toronto Research Ethics Board.

\section{Measures}

Participants provided demographic information consisting of age, sex, height and weight, living situation (e.g. on/off campus/parental home) and information on student status (e.g. full-time/part-time, year of study).

The eight health-risk behaviours measured were insufficient physical activity, fruit and vegetable intake, and sleep and smoking, marijuana use, illicit drug use, binge drinking and risky sexual behaviours. The five mental health outcomes measured were fatigue, stress, diagnosed depression, diagnosed anxiety and psychological distress.

Smoking (cigarettes), marijuana, illicit drugs As with Canadian surveillance, ${ }^{16}$ participants were asked about their cigarette smoking and marijuana and illicit drug use: "Within the last 30 days, on how many days did you use the following..." Response options ranged from "never used" to "have used but not in the last 30 days" to "used all 30 days." Responses were dichotomized to represent users (in the past 30 days) or non-users (have not used in the past 30 days).

\section{Binge drinking}

Participants were asked, "Within the last 15 days, how many times did you have 5 or more drinks in one sitting?" As in previous research, ${ }^{17}$ respondents who answered that they engaged in one or more sessions of binge drinking during 
TABLE 1

Participant and mental health outcome characteristics

\begin{tabular}{|c|c|c|c|}
\hline & $\begin{array}{c}\text { Overall } \\
(N=837)\end{array}$ & $\begin{array}{c}\text { Male } \\
(n=299)\end{array}$ & $\begin{array}{c}\text { Female } \\
(n=538)\end{array}$ \\
\hline Mean age (SD) & $21.00(4.25)$ & $21.43(5.76)$ & $20.75(3.10)$ \\
\hline \multicolumn{4}{|l|}{ Place of residence, $\mathbf{n}(\%)$} \\
\hline On-campus & 183 (21.9) & 71 (23.7) & $112(20.8)$ \\
\hline Off-campus & $654(78.0)$ & $228(76.3)$ & $426(79.2)$ \\
\hline \multicolumn{4}{|l|}{ Study year, n (\%) } \\
\hline First & 199 (23.8) & $69(23.1)$ & $130(24.2)$ \\
\hline Second & $228(27.2)$ & $85 \quad(28.4)$ & $143(26.6)$ \\
\hline Third & $196(23.4)$ & $72(24.1)$ & $124(23.0)$ \\
\hline Fourth & 162 (19.4) & $54(18.1)$ & $108(20.1)$ \\
\hline Fifth or higher & $52(6.2)$ & $19(6.4)$ & $33(6.1)$ \\
\hline \multicolumn{4}{|l|}{ Stress, n (\%) } \\
\hline Not stressed & $362(43.2)$ & $139(46.5)$ & $223(41.4)$ \\
\hline Stressed & $473(56.5)$ & 158 & $315(58.6)$ \\
\hline No response & $2(0.2)$ & $2(0.7)$ & \\
\hline \multicolumn{4}{|l|}{ Fatigue, n (\%) } \\
\hline Not fatigued & $453(54.1)$ & 160 & $293(54.5)$ \\
\hline Fatigued & $380(45.4)$ & $138(46.2)$ & $242(45.0)$ \\
\hline No response & $4(0.5)$ & $1(0.3)$ & $3(0.6)$ \\
\hline \multicolumn{4}{|l|}{ Diagnosed anxiety, n (\%) } \\
\hline No & 766 (91.5) & $281(94.0)$ & $485(90.1)$ \\
\hline Yes & 71 (8.5) & $18(6.0)$ & $53(9.9)$ \\
\hline \multicolumn{4}{|c|}{ Diagnosed or treated for depression, $n$ (\%) } \\
\hline No & 775 (92.6) & 283 (94.6) & $492(91.4)$ \\
\hline Yes & $62(7.4)$ & $16(5.4)$ & $46(8.6)$ \\
\hline Mean psychological distress (SD) & $3.55(2.64)$ & $3.04(2.60)$ & $3.90(0.24)$ \\
\hline
\end{tabular}

Abbreviation: SD, standard deviation.

Note: Place of residence, study year, stress, fatigue, diagnosed anxiety and diagnosed depression are represented by the number of participants (percentages); age and psychological distress reflect mean scores (standard deviations).

the past 15 days were considered binge drinkers, while those who did not engage in binge drinking within the past 15 days were classified as non-binge drinkers.

\section{Risky sexual behaviour}

Participants were asked, "Within the last 12 months, have you experienced the following as a consequences of your drinking ... had unprotected sex?" Responses were either yes or no (because they do not drink or they did not engage in this healthrisk behaviour). Consistent with previous research in this area, ${ }^{11}$ a positive response was considered as risky sexual behaviour.

\section{Insufficient physical activity}

Moderate-to-vigorous physical activity (MVPA) behaviours were assessed using two questions: "On how many of the past 7 days did you: Do moderate-intensity cardio or aerobic exercises (caused a noticeable increase in heart rate, such as brisk walk) for at least 30 minutes?" and whether they "[did] vigorous-intensity cardio or aerobic exercises ([that] caused large increase in breathing or heart rate such as jogging) for at least 20 minutes?" Participants chose answers from a scale of 0 to 7 days. Consistent with the previous Canadian physical activity guidelines, ${ }^{18}$ the scores of the two items were added and classified as "insufficiently active" (students who engaged in $<3$ days of MVPA per week) or "sufficiently active" (students who engaged in MVPA for $\geq 4$ days per week).
Insufficient fruit and vegetable intake

Participants were asked "How many servings of fruits and vegetables do you usually have per day? (1 serving $=1$ medium piece of fruit; $1 / 2$ cup fresh, frozen, or canned fruits/vegetables; $3 / 4$ cup fruit/vegetable juice; 1 cup salad greens; $1 / 4$ cup dried fruits)." The response options were 0 servings per day, 1 to 2 servings per day, 3 to 4 servings per day and 5 or more servings per day. Responses were dichotomized to reflect sufficient versus insufficient fruit and vegetable intake ( $\geq 5$ servings of fruit and vegetables each day versus $<5$ servings of fruits and vegetables). Although the consumption of 5 or more servings per day is below the Canadian recommendations, ${ }^{19}$ this measure was consistent with earlier US recommendations. ${ }^{20}$

\section{Insufficient sleep}

Participants were asked, "On how many of the past 7 days did you get enough sleep so you felt rested when you woke up in the morning?" Consistent with earlier research, ${ }^{11}$ responses were dichotomized to reflect either sufficient (restful on $\geq 4$ nights per week) or insufficient ( $<4$ nights) sleep on most nights of the week.

\section{Fatigue}

Participants were asked, "In the past 7 days, how often have you felt tired, dragged out, or sleepy during the day?" Participants were considered to have greater than normal fatigue if they reported 4 or more days, while those who reported less than 4 days were considered to have normal fatigue.

\section{Stress}

A single question asked, "Within the last 12 months, how would you rate the overall level of stress that you have experienced?" Participants who chose "no stress," "less than average stress" or "average stress" from the list were considered to have had normal stress, while those who chose "more than average stress" or "tremendous stress" were categorized as having greater than normal stress.

\section{Diagnosed depression and anxiety}

Participants were asked, "Within the last 12 months, have you been diagnosed or 
treated by a professional for any of the following?" Responses for depression and anxiety were each dichotomized as "yes" (yes, diagnosed but not treated; yes, treated with medication; yes, treated with psychotherapy; yes, treated with medication and psychotherapy; yes, other treatment) or "no" (have not been diagnosed or treated).

\section{Psychological distress}

Six questions were used to measure psychological distress. These questions asked participants, "Have you ever felt things were hopeless; felt overwhelmed by all you had to do; felt exhausted (not from physical activity); felt very lonely; felt very sad; felt overwhelming anxiety). Affirmative responses (i.e. yes, in the last 12 months) were summed and reported as a composite score of psychological distress.

\section{Statistical analysis}

We conducted LCA to identify the underlying patterns (or classes) of health-risk behaviours based on student responses to the questions on binge drinking, smoking, marijuana and illicit drug use, risky sexual behaviour, and insufficient physical activity, fruit and vegetable intake and sleep. To ensure that the maximum likelihood solution was correctly identified within these models, we conducted 200 iterations of each model (i.e. from two to four). We also randomly generated start values to ensure we achieved true maximum likelihood. Using Mplus version 6.1, ${ }^{21}$ decisions about the optimal number of latent classes were based on model fit statistics including Akaike Information Criterion (AIC); Bayesian Information Criterion (BIC); sample-size adjusted Bayesian Information Criterion (ABIC); entropy; and the Lo-Mendell-Rubin's likelihood ratio (LMR) and bootstrapped Lo-Mendell-Rubin's likelihood ratio tests (BLMR).22

To select the appropriate number of latent classes, we analyzed a two-class model and compared this analysis with successive models, which specified an increasing number of latent classes (up to four). We examined specific model estimates for both three- and four-class models to select a final specification based on the interpretability of the results. ${ }^{11}$ A test of measurement invariance revealed no significant sex differences between constrained and non-constrained models. Sex, year of study and place of residence (living on or off campus) were included as covariates in the final models. We used the auxiliary function in Mplus to examine the relationship between class membership and mental health outcomes of stress, fatigue, anxiety, depression and psychological distress in a multinomial logistic regression. Each mental health outcome was examined as a separate dependent variable, and test of equality of means across classes using posterior probability-based multiple imputations are reported.

\section{Results}

\section{Latent class findings}

Based on the eight health-risk behaviours of insufficient physical activity, insufficient fruit and vegetable intake, insufficient sleep, smoking, binge drinking, marijuana use, illicit drug use and risky sexual behaviours, all of which were adjusted for sex, year of study and place of residence (on or off campus), a three-class model of undergraduate students demonstrated the best model fit (see Table 2). Response probabilities for each class - the likelihood of engaging in each of the health-risk behaviours-are shown in Figure 1, and can be described as follows:

\section{Class 1: Typical students}

This group is characterized as the most common clustering of the modifiable health-risk behaviours, representing approximately two-thirds $(65.7 \%)$ of the sample. These students were unlikely to smoke or engage in sexual risk-taking and marijuana or illicit drug use (probabilities between $1.6 \%$ to $6.4 \%$ ), although they are also unlikely to engage in health-protecting behaviours. Probabilities of typical students eating enough fruits and vegetables $(6.2 \%)$, being physically active $(12.2 \%)$ and getting sufficient sleep (27.6\%) were low (see Figure 1). Of note, among the three classes identified, the typical students reported the lowest probabilities of binge drinking (35.7\%).

TABLE 2

Criteria to assess model fit

\begin{tabular}{|c|c|c|c|c|c|c|}
\hline & \multicolumn{3}{|c|}{ Model fit without covariates } & \multicolumn{3}{|c|}{ Model fit with covariates } \\
\hline & 2 Class & 3 Class & 4 Class & 2 Class & 3 Class & 4 Class \\
\hline Log likelihood & -2940.43 & -2913.89 & -2903.32 & -2924.82 & -2897.72 & -2880.59 \\
\hline BIC & 5995.27 & 6002.75 & 6042.17 & 5984.18 & 6010.71 & 6057.18 \\
\hline $\mathrm{ABIC}$ & 5941.29 & 5920.18 & 5931.36 & 5920.66 & 5909.09 & 5917.49 \\
\hline BLMR & $-3056.22^{*}$ & $-2940.43^{*}$ & -2913.89 & -3043.37 & $-2924.82^{*}$ & -2897.72 \\
\hline Estimated parameters & 17 & 26 & 35 & 20 & 32 & 44 \\
\hline
\end{tabular}

Abbreviations: ABIC, Adjusted Bayesian Information Criterion; AIC, Akaike Information Criterion; BIC, Bayesian Information Criterion; BLMR, Bootstrap Lo-Mendell-Rubin Test; LMR, Lo-Mendell-Rubin test.

${ }^{*} p<.05$. 
FIGURE 1

Item-response probabilities for health-risk behaviours across the three classes (typical, high-risk and moderately healthy) that result from latent class analysis of student behaviour

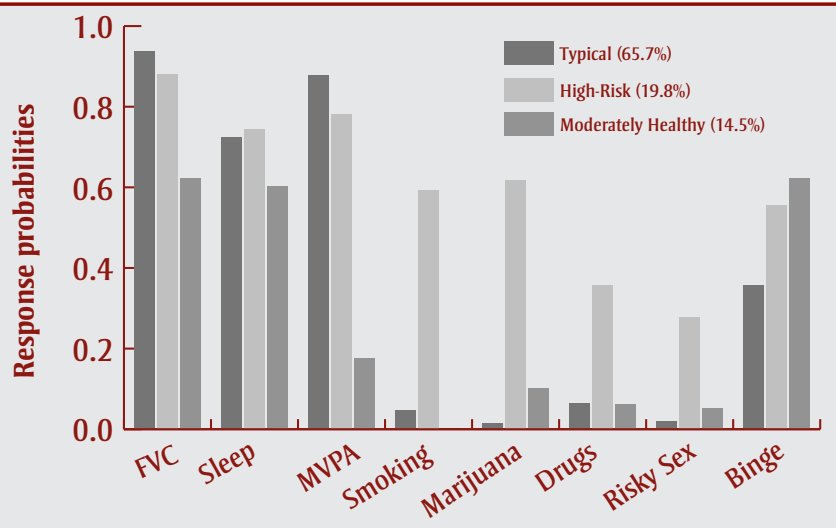

Health-risk behaviours

Abbreviations: FVC, fruit and vegetable consumption; MVPA, moderate-to-vigorous physical activity.

Notes: FVC, sleep and MVPA were reversed coded so that lower probabilities of engaging in these behaviours reflect higher risk. Typical students represent $65.7 \%$, high-risk students $19.8 \%$, and moderately healthy students $14.5 \%$ of the sample.

\section{Class 2: High-risk students}

This group can be characterized as exhibiting poor health behaviours across all of the eight behavioural domains. Like the typical student group, these students were unlikely to engage in health-protecting behaviours (i.e. probabilities of eating enough fruit and vegetable, being physically active and getting enough sleep were all less than $25 \%$ ). However, they demonstrated the highest likelihood of healthrisk behaviours. The probability of high-risk students smoking cigarettes, using marijuana and binge drinking ranged between $55.4 \%$ and $61.8 \%$, and their probability for using illicit drugs $(35.7 \%)$ and engaging in unprotected sex $(27.8 \%)$ was also the highest. This class represented approximately $20 \%$ of the student sample (see Figure 1).

\section{Class 3: Moderately healthy students}

This group, $14.5 \%$ of the sample, represents those students with the healthiest lifestyles. Similar to the typical student group, moderately healthy students were unlikely to smoke, engage in unprotected sex and marijuana or illicit drug use (probabilities from $0 \%$ for smoking to $6.3 \%$ for illicit drug use). Despite having the highest likelihood of consuming at least 5 servings of fruits or vegetables $(37.7 \%)$ and getting sufficient sleep (39.8\%), probabilities of engaging in these health-promoting behaviours were modest at best. Moderately healthy students, however, were highly likely to be physically active (with $82.4 \%$ of students meeting recommendations for MVPA), but also highly likely to binge drink (62.3\%) (see Figure 1).

\section{Class membership and mental health outcomes}

Overall, the differences in reported stress on the basis of class membership were significant $\left(\chi^{2}(2833)=8.55, p<.05\right)$. Specifically, students in the high-risk group reported significantly higher levels of stress than the typical class $\left(\chi^{2}(1671)=7.26, p<.01\right)$, but not compared to the moderately healthy $\left(\chi^{2}(1285)=3.12, p=.07\right)$ class of students. While non-significant, descriptive statistics show high-risk students reporting the highest prevalence of other mental health issues, including greater levels of self-reported fatigue, diagnosed anxiety, diagnosed depression and reported psychological distress. Conversely, moderately healthy students reported the lowest prevalence on most of the mental health issues examined. (See Table 3 for a complete description of the mental health outcomes based on class membership.)

\section{Discussion}

Our findings revealed several important and distinct patterns of health-risk behaviours in a sample of Canadian undergraduate students. Specifically, we found three discernable groups based on a shared health-risk behaviour profile. These ranged from students likely to engage in multiple health-risk behaviours such as smoking, binge drinking, drug use (marijuana and other illicit drugs) and risky sexual behaviours, to "typical" and "moderately healthy" students who generally did not engage in these risky behaviours. A troubling finding, however, is that the vast majority of students, regardless of class membership, had low probabilities of engaging in healthpromoting behaviours. In particular, each of the groups shared similar high probabilities of inadequate sleep and fruit and vegetable consumption.

Traditionally, public health has focussed on reducing health-risk behaviours, particularly those associated with noncommunicable diseases such as smoking or alcohol use. ${ }^{23}$ Current findings, however, highlight that the most prevalent health-risk behaviours on campus are those that students are not engaging in (i.e. physical activity, fruit and vegetable consumption and sufficient sleep). Even moderately healthy students were likely to be not getting enough sleep and consuming less than half of the nationally recommended 8 to 10 daily servings of fruits and vegetables. ${ }^{19}$ These behavioural patterns may reflect the difficulties (e.g. time needed to engage in physical activity; cost of and access to fruit and vegetables) for students in self-regulating complex behaviours such as physical activity, dietary intake and sleep during this major life transition.

These behavioural patterns are also of concern given that evidence shows that smoking, binge drinking and insufficient physical activity increase throughout adolescence and into early adulthood.,24 While young adults tend to stop binge drinking and smoking once they reach their mid-twenties, patterns of physical inactivity only continue to worsen. ${ }^{4}$ This 
TABLE 3

Comparison of mental health outcomes by class membership based on equality test of means across classes

\begin{tabular}{|c|c|c|c|c|}
\hline \multirow[b]{3}{*}{ Stress } & \multicolumn{4}{|c|}{$\begin{array}{l}\text { Probability of mental health problems } \\
\qquad \%(\mathrm{SE})\end{array}$} \\
\hline & \multirow{2}{*}{$\begin{array}{c}\begin{array}{c}\text { Overall } \\
(N=837)\end{array} \\
56.5^{*}\end{array}$} & \multirow{2}{*}{$\begin{array}{c}\begin{array}{c}\text { Typical } \\
(65.7 \%)\end{array} \\
54.1 \quad(2.2)^{\mathrm{a}}\end{array}$} & $\begin{array}{l}\text { High risk } \\
(\mathbf{1 9 . 8 \% )}\end{array}$ & $\begin{array}{c}\text { Moderately healthy } \\
(\mathbf{1 4 . 5 \% )}\end{array}$ \\
\hline & & & $66.9(4.0)$ & $54.5(5.3)^{* *}$ \\
\hline Fatigue & 45.4 & $45.9(2.3)$ & $49.9 \quad(4.4)$ & $39.0 \quad(5.5)^{* *}$ \\
\hline Diagnosed anxiety & 8.5 & $7.3(1.2)$ & $11.2 \quad(2.7)$ & $9.5 \quad(3.2)$ \\
\hline Diagnosed depression & 7.4 & $6.7(1.1)$ & $9.9 \quad(2.5)$ & $6.5 \quad(2.7)$ \\
\hline Psychological distress & $\begin{array}{c}3.55 \\
(+2.64)\end{array}$ & $3.52(0.12)$ & $3.90 \quad(0.24)$ & $3.26(0.28)^{* *}$ \\
\hline
\end{tabular}

Abbreviation: SE, standard error.

Note: With the exception of psychological distress, prevalence statistics are represented as percentages; scores for psychological distress range from $0-6$; sample sizes are estimated based on probability of latent class membership within classes.

${ }^{a}$ Significantly different from high-risk class.

${ }^{*} p<.05$.

${ }^{* *} p<.09$.

body of evidence highlights the need to reorient health services to ensure health promotion efforts address the lack of health-promoting behaviours as well as the more commonly targeted health-risk behaviours such as smoking and drug use. Initiatives such as Healthy Campus 2020 framework ${ }^{25}$ have been strong proponents for increasing targeting of diet and physical activity among young adults but, to achieve these goals, more campus initiatives aimed at promoting healthy active living are required.

Interestingly, with the exception of binge drinking, there were distinctions between classes in how they engaged in common health-risk behaviours. The overall prevalence of these health-risk behaviours was fairly low, although a clustering of students appeared to be engaging in many of these risky behaviours. These findings suggest that current health promotion strategies are generally effective for a majority of the undergraduate student population but that an at-risk group may require tailored intervention. This intervention may take the form of health promotion materials targeting these health-risk behaviours together rather than in isolation. By targeting multiple health-risk behaviours simultaneously, individuals may be able to transfer their knowledge and experiences from one behaviour to another if the domains share similarities. ${ }^{26}$ The idea is to be able to prompt change in one of these behaviours, which may result in a cascading effect, similar to previous research showing a corresponding decrease in marijuana and alcohol use when quitting smoking. ${ }^{26}$

\section{Multiple health-risk behaviours and mental health outcomes}

Our study also examined how these patterns of multiple health-risk behaviours were associated with mental health. Broadly, our findings support the notion that engaging in multiple health-risk behaviours is associated with poorer mental health. Although the results were not statistically significant, moderately healthy students reported less fatigue, stress and psychological distress than high-risk students. These are important findings, as psychological distress is often considered a robust indicator of health, ${ }^{27}$ while management of stress and fatigue is crucial for academic success. ${ }^{28}$ Future research must examine these associations over time, as our cross-sectional study cannot determine the direction of the associations. However, given that student stress is an important focus for the Healthy Campus 2020 framework, ${ }^{25}$ interventions do need to be developed to jointly target health-risk behaviours and stress. Promoting physical activity as a way to cope with stress is an example of an intervention approach that targets mental and physical health. Future research that focusses on the at-risk student population will also be important, as their health-risk behaviour profile may reflect maladaptive coping strategies. Potential interventions may be aimed at replacing harmful coping strategies such as smoking and drinking with healthy coping strategies such as regular sleep and physical activity.

\section{Strengths and limitations}

While our study uses a fairly novel statistical technique to examine the clustering of multiple health-risk behaviours in a 
cohort of university students, we need to acknowledge several limitations. First, there is an obvious response bias, given that only $28 \%$ of the undergraduate students invited to participate in the survey responded. Although the low response rate is comparable to other studies that used NCHA data in the United States $^{30}$ and Canada,,6 our findings nevertheless need to be interpreted with caution.

Second, these data included students from a single university located in a large urban setting. Although the sample was broadly representative of the undergraduate student body at the University of Toronto (i.e. median age $=21.1$ years, $56 \%$ female), ${ }^{29}$ we are limited in our abilities to generalize these findings to students in other postsecondary institutions.

Third, while self-report tools such as the NCHA are critical for gathering health data, analyzing secondary data has its limitations. For example, we were limited by the measures included in the survey and did not have validated measures of mental health outcomes such as anxiety and depression. Moreover, we examined health-risk behaviours assessed on different time scales (i.e. past 7 days, 15 days or 30 days). Although the measures with longer time scales were intended to capture the less common health-risk behaviours (i.e. illicit drug use), it would be ideal to have consistency across each of the behaviours.

Finally, while our analyses included sex, student status and place of residence as covariates, other sociodemographic factors may be important in differentiating class membership. Future research could develop more descriptive profiles of each class in order to assist in tailoring interventions. Future work that uses longitudinal designs to assess the same students over time is also required to determine the direction in the relationship between health-risk behaviours and outcomes such as mental health.

\section{Conclusion}

The transition into early adulthood is an important target for health promotion efforts, and the postsecondary campus is an ideal setting for comprehensively addressing the health of many young adults. Responding to the call for action of the Okanagan Charter, ${ }^{2}$ our findings reinforce the need to consider what health behaviours campus health services are targeting, acknowledging that a greater emphasis should be placed on supporting health-protecting behaviours of diet, physical activity and sleep. The caveat is that health promoters will need to be mindful of the collective challenges students face and the complexities of behaviour change, and recognize that changes in lifestyle behaviours will likely need to be accompanied by the development of selfregulation skills to manage their competing interests while at college or university. Overall, our findings suggest that patterns of these multiple health behaviours are related to mental health in the postsecondary student population, but more research examining the impact of multiple health behaviours over time is needed.

\section{Acknowledgements}

Guy Faulkner is a Chair in Applied Public Health funded by the Public Health Agency of Canada (PHAC) in partnership with the Canadian Institutes of Health Research (CIHR).

We would like to acknowledge the support of the Canadian Organization of University and College Health for their support on this project.

\section{References}

1. Association of Universities and Colleges of Canada. Trends in higher education. Volume 1-Enrolment [Internet]. Ottawa (ON): AUCC; 2011 [cited 2013 Jun 24]. Available from: http://www.cais.ca/ uploaded/trends-2011-vol1-enrolment-e.pdf

2. The Okanagan Charter for Health Promoting University and Colleges. Working draft: a shared vision for health promoting universities and colleges [Internet]. Kelowna (BC): Okanagan Charter; 2015 Jun 22 [cited 2015 Aug 18]. Available from: http://internationalhealthycampuses 2015 .sites.olt.ubc.ca/files/2015/06/Charter -Draft-One-Final-June-19-2015.pdf
3. Gall TL, Evans DR, Bellerose S. Transition to first-year university: patterns of change in adjustment across life domains and time. J Soc Clin Psychol. 2000;19:544-67. doi: 10.1521/jscp.2000.19.4.544.

4. Kwan MY, Cairney J, Faulkner GE, Pullenayegum EE. Physical activity and other health-risk behaviours during the transition into early adulthood. Am J Prev Med. 2012;42:14-20. doi: 10.1016/j. amepre.2011.08.026.

5. Kwan MY, Faulkner GE, ArbourNicitopoulos KP, Cairney J. Prevalence of health-risk behaviours among Canadian post-secondary students: descriptive results from the National College Health Assessment. BMC Pub Health. 2013;13:548. doi: 10.1186/1471-2458-13-548.

6. Arbour-Nicitopoulos KP, Kwan MY, Taman S, Lowe D, Faulkner GE. Normative beliefs in health behavioural practices in a college population. J Am Coll Health. 2011;59:191-6.

7. Bray SR Kwan MYW. Physical activity is associated with better health and psychological well-being during transition to university. J Am Coll Health. 2006; 55: 78-82.

8. Jackson CA, Henderson M, Frank JW, Haw SJ. An overview of prevention of multiple risk behaviour in adolescence and young adulthood. J Pub Health. 2012; 34 Suppl 1:i31-40. doi: 10.1093/pubmed/ fdr113.

9. Slater MD, Flora JA. Health lifestyles: audience segmentation analysis for public health interventions. Health Educ Behav. 1991;18:221-33.

10. Hagenaars JA, McCutcheons AL. (Eds.). Applied latent class analysis. Cambridge (UK): Cambridge University Press; 2002.

11. Laska MN, Pasch KE, Lust K, Story $\mathrm{M}$, Ehlinger E. Latent class analysis of lifestyle characteristics and health risk behaviours among college youth. Prev Sci. 2009;10(4):376-86. doi: 10.1007/ s11121-009-0140-2.

12. Alexander SJ, Harrison AG. Cognitive responses to stress, depression, and anxiety and their relationship to ADHD symptoms in first year psychology students. J Atten Dis. 2013;17:29-37 
13. Stallman HM. Psychological distress in university students: a comparison with general population data. Aust Psychol. 2010; 45: 249-57.

14. American College Health Association (ACHA). American College Health Association - National College Health Assessment II: Reference Group Executive Summary Fall 2009. Linthicum (MD): American College Health Association; 2009.

15. American College Health Association The American College Health Association National College Health Assessment (ACHA-NCHA), Spring 2005 Reference Group Data (Abridged). J Am Coll Health. 2006;55:5-16.

16. DeRuiter WK, Cairney J, Leatherdale S, Faulkner G. The period prevalence of risk behaviour co-occurrence among Canadians. Prev Med. 2016;85:11-6. doi:10.1016/j.ypmed.2015.11.026.

17. Nelson MC, Lust K, Story M, Ehlinger E. Alcohol use, eating patterns and weight behaviours in a university population. Am J Health Beh. 2009;33:227-37.

18. Public Health Agency of Canada. Physical activity guidelines. Canada's physical activity guides [Internet]. Ottawa (ON): Public Health Agency of Canada; 2006 [cited 2012 Aug 1, guides no longer available]. Information available at: http://www.phac-aspc.gc.ca/hp-ps/ hl-mvs/pa-ap/03paap-eng.php

19. Health Canada. Eating well with Canada's food guide [Internet]. Ottawa (ON): Health Canada; 2011 [cited 2012 June 20]. Available from: http://www.hc-sc.gc.ca/fn-an/food -guide-aliment/gen_prin-eng.php

20. US Department of Health and Human Services. Dietary guidelines for Americans, 8th ed. Washington (DC): US Department of Health and Human Services; 2005. Joint publication of the US Department of Agriculture.

21. Muthén LK, Muthén BO. Latent variable hybrids: overview of old and new models. In Hancock GR, Samuelsen KM., editors. Advances in latent variable mixture models, pp. 1-24. Charlotte (NC): Information Age Publishing, Inc.; 2010.
22. Das P, Horton R. Rethinking our approach to physical activity. Lancet. 2012;380(9838):189-90. doi: 10.1016/ S0140-6736(12)61024-1.

23. Wing Kwan MY, Bray SR, Martin Ginis KA. Predicting physical activity during transition to first-year university: an application of the Theory of Planned Behaviour. J Am Coll Health. 2009;58:4552. doi: 10.3200/JACH.58.1.45-55.

24. Healthy People 2020 [Internet]. Washington (DC): US Department of Health and Human Services; [cited 2012 May]. Available from: http://www.healthypeople.gov

25. Barnett SM, Ceci SJ. When and where do we apply what we learn? A taxonomy for far transfer. Psychol Bull. 2002;128:612-37.

26. Adlaf EM, Demers A, Gliksman L, editors. Canadian campus survey 2004. Toronto (ON): Centre for Addiction and Mental Health; 2005.

27. Steptoe A, Tsuda A, Tanaka Y, Wardle J. Depressive symptoms, socio-economic background, sense of control, and cultural factors in university students from 23 countries. Int $\mathrm{J}$ Behav Med. 2007;14:97-102.

28. Nagane M. Relationship of subjective chronic fatigue to academic performance. Psychol Rep. 2004;95:48-52.

29. Keith DR, Hart CL, McNeil MP, Silver R, Goodwin RD. Frequent marijuana use, binge drinking and mental health problems among undergraduates. Am J Addict. 2015;24(6):499-506. doi: 10.1111/ ajad.12201.

30. Pask-Aubé C, Liang X. University of Toronto Facts and Figures 2006. Toronto (ON): University of Toronto, Office of the Vice-Provost, Planning and Budget; 2006. 